\section{Session 3: Cancer}

Monday 7th November 2011. Moderator: Yan Wu
[14.00-14.30]

'Advances in cancer immunotherapy'

Zdenka K. Jonak

GlaxoSmithKline, King-of-Prussia, PA, USA

Abstract not provided.

[14.30-15.00]

'Targeting in TGFBRII oncology: A potent immunomodulatory antibody with various effects on cancer progression'

John Haurum

Imclone Systems, New York, NY, USA

Signaling via the TGF $\beta$ pathway plays diverse roles in tumor progression, directly and indirectly driving tumor cell growth and pathogenesis. Direct pro-tumor effects, which occur in TGF $\beta$ receptor-expressing tumor cells, include autocrine mitogen production, epithelial to mesenchymal transition (EMT), invasion, migration, and prometastatic cytokine production. Indirect pro-tumor effects, which occur in stromal cells, include immunosuppression and angiogenesis. Due to the pleiotropic effects of the TGF $\beta$ pathway in cancer, we set out to disrupt signaling using a mAb that blocks the ectodomain of TGF $\beta$ RII, in order to inhibit receptor mediated signaling in target cells. Here we describe a high affinity, fully human anti-TGF $\beta$ RII mAb along with its murine surrogate. We observed in vivo efficacy in various human and murine tumor models treated with anti-TGF $\beta$ RII as a monotherapy. In addition, antiTGF $\beta$ RII therapy functioned at least additively with the cytotoxic cyclophosphamide. Using immune competent murine models of aggressive disease, we have shown that the major indirect mechanism of action of our antibody involves enhanced anti-tumor immunity. Specifically, using an immune-depletion strategy, we have shown that depletion of CD8+ cytotoxic T lymphocytes (CTL) eliminated the ability of anti-TGF $\beta$ RII to inhibit primary, but not metastatic, tumor growth. Conversely, depletion of NK cells eliminated the ability of anti-TGF $\beta$ RII to inhibit metastasis while having little effect on inhibition of primary tumor growth. Analysis of NK cells and CTL ex vivo showed that treatment with anti-TGF $\beta$ RII significantly induced killing activity by these two populations of cells. In addition, the Th1 cytokine response marked by IFN $\gamma$ secretion by NK cells and CTL was increased in anti-TGF $\beta$ RII treated animals. Therefore, antibody treatment significantly induced anti-tumor immunity in vivo. Finally, the circulating immunosuppressive $\mathrm{T}$ regulatory (Treg) and MDSC populations were reduced in anti$\mathrm{TGF} \beta$ RII treated animals. In vitro, treatment with the anti-TGF $\beta$ RII antibody inhibited TGF $\beta$-induced conversion of naive $\mathrm{T}$ cells into Treg cells and Treg cell mediated inhibition of $\mathrm{T}$ cell proliferation. Collectively, these data demonstrate that selective blockade of TGF $\beta$ RII with a neutralizing antibody suppresses primary tumor growth and metastasis through both direct and indirect attenuation of TGF $\beta$ signaling. The results of these studies provide compelling data supporting the utilization of a neutralizing anti-TGF $\beta$ RII antibody as a novel therapeutic strategy for the treatment of TGF $\beta$ dependent tumors. 
[15.00-15.20]

'Recombinant antibody mixtures for the treatment of cancer'

Torben P. Frandsen

Symphogen A/S, Copenhagen, Denmark

Abstract not provided.

[15.20-15.40]

'Antibody-Maytansinoid Conjugates (AMCs): realizing the promise of antibody-directed targeting in cancer patients'

John M. Lambert

ImmunoGen Inc., Waltham, Massachusetts, USA

Abstract not provided.

[15.40-16.00]

'Cytotoxicity of human antibody light chains against cancer cells'

Emi Hifumi and Taizo Uda

Oita University, Oita-shi, Japan

The authors have reported in HAH2009 about the preparation and expression of human catalytic antibody light chains possessing catalytic activity (amidase activity) which were mostly encoded in Subgroup II in kappa type by examining over 50 kinds of human antibody light chains. The study has substantially advanced for these two years. The highly purified human light chains were submitted to investigate the function whether they show the toxicity against cancer cells. We will introduce the interesting features of the human light chains prepared in this study.

We designed two primer sets for this purpose and performed a semi-nested PCR using cDNA prepared from human leukocyte as a template. As the result, eighteen clones of subgroup II germline gene of human kappa light chain were established. All clones were belonging to subgroup II. The 18 clones and some mutants of the clones were expressed in E.coli and the light chains recovered were highly purified by employing two-step purification system. All clones were investigated from the viewpoint of cytotoxicity against cancer cells. Out of them, \#1 clone (belonging to A18b germline gene) was unique. It showed the cytotoxicity to both human lung (A548 purchased from ATCC) and human stomach cancer cells (SNU-1) but not to pancreas cancer cells (PANC-1). Interestingly, the dimeric form showed the stronger effect than the monomeric form, which was prepared by the mutation of the wild type (C220A). The \#1 clone digested QAR-MCA synthetic peptide substrate, showing the amidase activity. The germline gene $\mathrm{A} 18 \mathrm{~b}$ possesses a catalytic triad-like structure composed of Ser, His and Asp by molecular modeling. In addition, \#1 clone has a unique amino acid sequence compared with that of other clones. Although it is still unclear that the relationship between catalytic activity and cytotoxicity of human light chain (\#1 clone) at this moment, the detail results and the assumed mechanism will be mentioned in the presentation. 